\title{
Histopatholgy and Clinical Implication of Treatment- Related Changes After Gamma Knife Radiosurgery in Patients With Brain Metastases
}

\section{Jeong-Hwa Kim}

Samsung Medical Center, Sungkyunkwan University School of Medicine Jung-Won Choi

Samsung Medical Center, Sungkyunkwan University School of Medicine Doo-Sik Kong

Samsung Medical Center, Sungkyunkwan University School of Medicine Ho-Jun Seol

Samsung Medical Center, Sungkyunkwan University School of Medicine

\section{Do-Hyun Nam}

Samsung Medical Center, Sungkyunkwan University School of Medicine

\section{Yeon-Lim Suh}

Samsung Medical Center, Sungkyunkwan University School of Medicine Jung-II Lee ( $\nabla$ jilee.lee@samsung.com )

Samsung Medical Center, Sungkyunkwan University School of Medicine Jae-Wook Ryu

Samsung Medical Center, Sungkyunkwan University School of Medicine Sung-Tae Kim

Samsung Medical Center, Sungkyunkwan University School of Medicine

\section{Research Article}

Keywords: treatment-related changes (TRCs), stereotactic radiosurgery (SRS), magnetic resonance imaging (MRI), Brain metastasis (BM)

Posted Date: June 21st, 2021

DOl: https://doi.org/10.21203/rs.3.rs-613147/v1

License: (1) (1) This work is licensed under a Creative Commons Attribution 4.0 International License. Read Full License 


\section{Histopathology and clinical implication of treatment-related changes after gamma knife radiosurgery in patients with brain metastases}

Jeong Hwa Kim, MD ${ }^{1}$ (https://orcid.org/0000-0002-8587-8546), Jung Won Choi, MD, PhD ${ }^{1}$, Doo-Sik Kong, $\mathrm{MD}, \mathrm{PhD}^{1}$ (https://orcid.org/0000-0002-7519-3594), Ho Jun Seol, $\mathrm{PhD}^{1}$

(https://orcid.org/0000-0003-4187-054X), Do-Hyun Nam, PhD ${ }^{1}$, Jae-Wook Ryu², Sung-Tae Kim² (https://orcid.org/0000-0001-8185-0063), Yeon-Lim Suh, $\mathrm{PhD}^{3}$ (https://orcid.org/0000-0001-58092401) and Jung-Il Lee*, $\mathrm{PhD}^{1}$ (https://orcid.org/0000-0001-8143-5513)

${ }^{1}$ Department of Neurosurgery, Samsung Medical Center, Sungkyunkwan University School of Medicine, Seoul, South Korea

${ }^{2}$ Department of Radiology, Samsung Medical Center, Sungkyunkwan University School of Medicine, Seoul, South Korea

${ }^{3}$ Department of Pathology, Samsung Medical Center, Sungkyunkwan University School of Medicine, Seoul, South Korea

*Corresponding Author: Jung-II Lee, MD, PhD

Department of Neurosurgery, Samsung Medical Center

Sungkyunkwan University School of Medicine

81 Irwon-ro, Gangnam-gu, Seoul 06351, South Korea

Phone: 82-2-3410-3499

Mobile phone: 82-10-9933-3494

E-mail: jilee.lee@samsung.com

ORCID: https://orcid.org/0000-0001-8143-5513 


\section{Abstract}

A late-onset treatment-related changes (TRCs), which represent radiographic radiation necrosis $(\mathrm{RN})$, frequently occur after stereotactic radiosurgery (SRS) for brain metastases and often need surgical treatment. This study aimed to validate the true pathology and investigate clinical implication of surgically resected TRCs on advanced magnetic resonance imaging (MRI).

Retrospective analyses of 86 patients who underwent surgical resection after radiosurgery of brain metastases were performed. Fifty-four patients displayed TRCs on preoperative MRI, comprising pure RN in 19 patients (TRC-RN group) and mixed viable tumor cells in 35 patients (TRC-PD group). Thirty-two patients revealed the consistent diagnosis of progressive disease in both MRI and histopathology (PD-PD group). The TRC-PD group showed larger prescription isodose volume $(9.4$ $\left.\mathrm{cm}^{3}\right)$ than the TRC-RN $\left(4.06 \mathrm{~cm}^{3}, \mathrm{p}=0.014\right)$ group and a shorter time interval from SRS to preoperative MRI diagnosis (median 4.07 months) than the PD-PD group (median 8.77 months, $\mathrm{p}=0.004)$. Progression-free survival was significantly different among the three groups $(\mathrm{p}<0.001)$, but not between TRC-RN and TRC-PD (post hoc test, $\mathrm{p}=1.00$ ), while no difference was observed in overall survival $(\mathrm{p}=0.067)$.

Brain metastases featured as TRCs after SRS frequently contained viable tumor cells. However, this histologic heterogeneity had a minor impact on benign prognosis of TRCs after surgical resection. 


\section{Introduction}

Brain metastasis (BM) is the most common and critical complication of systemic cancer. With the improvement of primary site control in solid tumors using chemotherapy and immunotherapy, management of metastatic lesions has become a major concern for patients with cancer ${ }^{1,2}$. Current guidelines for management of BMs include stereotactic radiosurgery (SRS) or radiotherapy as a key modality for local control with high efficacy ${ }^{3-5}$.

Of the various radiologic responses after radiation, differentiating treatment-related changes (TRCs) and progressive disease (PD) has been a matter of debate. Both present similar imaging appearance in conventional magnetic resonance imaging (MRI) of enlarged, heterogeneous rim enhancement in the T1-weighted sequence ${ }^{6,7}$. Although several advanced imaging reports such as perfusion or diffusion weighted, MR spectroscopy, and radiomics-oriented studies tried to discriminate TRCs from PD, they are mostly without histologic validation and remote from clinical application ${ }^{8-10}$.

The exact histological characteristics differentiating various TRCs have not been defined yet. Instead, physicians categorize different TRCs according to their onset time after radiation. Acute radiation injuries represent transient, reversible neurotoxic phenomena that occur within days to weeks, whereas early-delayed types appear weeks to several months following radiation. By contrast, $\mathrm{RN}$ is a late-delayed type of radiation injury observed $>3-6$ months post-radiation, with a frequently irreversible and progressive course ${ }^{11-14}$.

The clinical presentation of RN, a late-onset TRC, is highly variable. Some patients have no neurologic deficits, while symptomatic focal brain necroses occur in up to $10 \%$ of patients who undergo gamma knife radiosurgery (GKS) for BM. Indeed, the overall risk of RN leading to permanent morbidity and additional surgical resections was reported as $4.7-7.0 \%{ }^{4,5,15}$.

There is no standard treatment algorithm for RN, as the pathophysiology is not fully understood; thus, current management of RN mainly focuses on control of RN-related symptoms. Steroids or the 
vascular endothelial growth factor-A monoclonal antibody bevacizumab (Avastin) are promising to relieve the neurologic deficit, although long term use of these non-invasive medications is limited due to their complications, and patients eventually need surgical decompression ${ }^{16,17}$. However, few studies reported the surgical outcome and prognosis after resection of RN presenting as TRCs.

In this study, authors aimed to investigate the true histology and clinical prognosis after resection of late-onset TRCs. In this study, we defined "TRC" as a radiographic and symptomatic RN. Acute or early-delayed radiation injury and asymptomatic RN were excluded from data analyses because surgical treatment is not indicated for them. We presumed that several histological characteristics of TRCs may be the mixture of viable tumor cells with RN rather than pure necrosis. To evaluate the clinical implication of these "hiding" tumor cells that appear as TRCs, we included the radiographic PD group, comprising metastatic tumor cells in histology, and compared the prognosis of the TRC and PD groups.

\section{Results}

\section{Patient classification}

A total of 86 patients was included in the final analysis. Based on the radiological and histopathological diagnoses, patients were classified into three groups (Fig. 1). According to preoperative imaging, TRCs were diagnosed in 54 (62.7\%) patients and PD in $32(37.3 \%)$ patients. Among the patients with TRCs, the surgical pathology confirmed pure necrosis in only 19 of the 54 (35.2\%) patients whereas the other $35(64.8 \%)$ patients revealed mixed (11 of $35,31.5 \%)$ or pure metastatic ( 24 of $35,68.5 \%$ ) histology, represented as the TRC-RN and TRC-PD groups, respectively. All 32 patients with MRI-diagnosed tumor progression were histopathologically confirmed as having tumor progression and included in the PD-PD group. 


\section{Patient demographics and lesion characteristics}

The preoperative patient characteristics and treatment parameters are shown in Table 1. The median age of the entire study population was 58 years (range: $40-81$ years). Most metastatic lesions were in the frontal (40 patients, $46.5 \%$ ) and parietal (22 patients, $25.6 \%)$ regions with similar proportions in all three groups $(\mathrm{p}=0.754)$. Sixteen patients underwent fractionated radiosurgery due to a prolonged beam-on-time of $>2 \mathrm{~h}$. There was a mean isodose of $24 \mathrm{~Gy}$ in three fractions for 14 patients, and 18 Gy in two fractions and 30 Gy in four fractions for one patient each.

There were significant differences in multiple GKS history especially for the surgically resected lesions between the three groups $(\mathrm{p}=0.005)$. A much higher percentage of patients $(63.1 \%)$ in the TRC -RN group had a history of multiple GKS for the resected metastatic lesion, whereas nine $(25.7 \%)$ had it in the TRC-PD group and seven (21.6\%) in the PD-PD group (Jonckheere-Terpstra test, $\mathrm{z}=-2.68$, $\mathrm{p}=0.007)$. In contrast, there was no significant difference in the proportions of history of whole brain radiation therapy $(\mathrm{p}=0.117)$.

The median prescription isodose volume (PIV) was $6.41 \mathrm{~cm}^{3}$ (range: $0.14-62.29 \mathrm{~cm}^{3}$ ). Statistical differences were observed among the median PIVs of the three groups ( $\mathrm{p}=0.014)$, and the median PIV of the TRC-RN group $\left(4.06 \mathrm{~cm}^{3}\right.$, range $\left.0.14-34.50 \mathrm{~cm}^{3}\right)$ was smaller than that of the TRC-PD group $\left(9.40 \mathrm{~cm}^{3}\right.$, range $\left.1.50-40.72 \mathrm{~cm}^{3}\right)$ in the post hoc test $(\mathrm{p}=0.011)$. Radiation doses were delivered to $50 \%$ isodose line (IDL) in all patients with a median prescription dose of $18 \mathrm{~Gy}$ (range: 8-25 Gy).

The median time from the date of GKS to the imaging diagnosis was 6.07 months (range: $1.01-$ 40.03 months). Upon post hoc analysis, the TRC -PD group exhibited a shorter time to imaging diagnosis than the PD-PD group (4.07 months, [range: 1.03-18.13 months] vs. 8.77 months [range: $1.03-40.03$ months], respectively; $\mathrm{p}<0.001)$.

\section{Surgical outcomes and salvage therapy}

81 patients (94\%) underwent surgical resection within four weeks and the other five patients within 6 weeks after the time of radiologic diagnosis. Preoperative neurological deficits were headache in 57, motor weakness in 27 , nausea and vomiting in 16 , seizure in 13 , and aphasia in 10 patients. The 
median preoperative KPS score of the patients was 70 (range: 60-90). Total resection of the lesions was conducted in most of the patients to relieve the cerebral mass effect and promote better local control, except two patients in the PD-PD group underwent subtotal lesionectomy due to a poorly demarcated tumor related to irradiation or adherence to an anatomically critical structure such as the anterior clinoid process.

The median postoperative KPS score was 80 (range: 50-90), which was better than the preoperative KPS score in all three groups. The neurological deficits improved for most patients $(66,76.6 \%)$ but were sustained or worse after surgery in the other 20 (23.3\%) patients (Table 2). No major intraoperative complications were observed. One patient had newly developed hemiplegia in the immediate postoperative status, a predictable complication due to the location of the tumor in the thalamus.

After surgical resection, the chemotherapy regimen was changed according to disease progression status in the TRC-PD and PD-PD groups. The TRC-RN group was assessed as having stable disease and maintained the regimen. Postoperative GKS for the tumor bed was applied in only two patients in PD-PD group due to subtotal removal of the PD lesion, within a month after surgery. Other patients with total removal of metastatic lesions were clinically followed-up without any adjuvant local therapy and additional radiosurgery or surgical resection was performed only if local or distant progression was confirmed in radiologic evaluation.

\section{Survival analysis}

The median overall survival (OS) for the 86 patients was 21.7 months (95\% confidence interval [CI]: 12.9-30.6 months). The Kaplan-Meier estimates and median OS of each group are shown in Fig.

2. Patients in the TRC-RN group displayed a tendency toward longer survival than patients in the pathologic PD group: 1-year and 2-year actuarial survival was $82.4 \%$ and $75.5 \%$, respectively, in the TRC-RN group, compared with $63.2 \%$ and $41.5 \%$ in the TRC-PD and $72.9 \%$ and $27.5 \%$ in the PDPD groups, respectively, although statistical significance was not observed $(\mathrm{p}=0.067)$. 
confirmed using diagnostic MRI in $7(36 \%), 11(47 \%)$, and 23 (71\%) patients in the TRC-RN, TRCPD, and PD-PD groups, respectively. The median progression-free survival (PFS) was obtained for the PD-PD group alone (5.4 months [95\% CI: $2.7-8.0$ months]). Less than $50 \%$ of patients of other two group were confirmed of local progression during the data collection period, thus median PFS were not calculated.

There was a significant difference in PFS among the three groups (Fig. 3, p<0.0001). In the post hoc test, the PD-PD group exhibited a shorter time to progression than the other two groups $(\mathrm{p}=0.001$ vs. TRC-RN and $\mathrm{p}=0.002$ vs. TRC-PD), whereas no difference was observed between the TRC-RN and TRC-PD groups (p=1.0) (Fig. 3).

\section{Subgroup analysis of TRC-PD group}

For details of histopathological investigation of clinical prognosis, we further stratified the TRC-PD group according to its surgical pathology, as "TRC-Pure PD" including metastatic tumor cells alone (24 patients) and "TRC-Mixed PD/RN" including mixed pathology of radiation necrosis and viable tumor cells (11 patients). The Kaplan-Meier curve and log-rank tests revealed no significant difference between two subgroups of patients with TRC-PD either for OS or local progression (Fig. $4 a, b)$. These results suggest that the histopathologic heterogeneity of TRCs did not have influence on the clinical prognosis of metastatic lesions after surgical resection.

\section{Prognostic factors for survival and disease progression}

The results of the univariate and multivariate analyses according to clinical variables of patient groups are shown in Table 2. Clinical variables with $p$-values $<0.1$ in the univariate analyses were entered into the multivariate analysis using the Cox proportional-hazards model.

In the univariate and multivariate analyses, a significant association was observed between the radio-surgical pathologic diagnoses and lesion progression $(\mathrm{p}=0.0002$ and $\mathrm{p}=0.0016$ in the univariate and multivariate analyses, respectively); there was a higher risk of recurrence in the PD-PD group, with a hazard ratio (HR) of 4.11 (95\% CI: $1.73-9.79, \mathrm{p}=0.0002)$ in the univariate analysis and an HR 
of $5.46(95 \% \mathrm{CI}: 1.86-16.06, \mathrm{p}=0.002)$ in the multivariate analysis, compared with that in the TRC $\mathrm{RN}$ group (HR=1, reference). The risk of progression was not significantly different between the TRC-RN and TRC-PD groups ( $\mathrm{p}=0.535)$ (Table 2$)$.

Uncontrolled primary malignancy was associated with a significantly higher risk of death than controlled primary malignancy in the univariate analysis (HR: 2.027 [95\% CI: 1.143-3.595], $\mathrm{p}=0.020)$, and there was no association in the multivariate analysis $(\mathrm{p}=0.13)$. Primary malignancy showed a trend toward a higher risk of death in the univariate analysis $(\mathrm{p}=0.056)$. The other cancer groups exhibited an HR of 2.305 (95\% CI: 1.165-4.559, $\mathrm{p}=0.02)$ compared with the lung cancer group, although there was no significant association in the multivariate analysis $(\mathrm{p}=0.22)$. A higher prescription isodose (Gy) was associated with a significantly decreased risk of local progression in the univariate analysis alone (HR: 0.887 [95\% CI 0.796-0.989], $\mathrm{p}=0.030$ ) but not in the multivariate analysis $(\mathrm{p}=0.25)$. For primary malignancy, breast cancer and other types of cancer exhibited a higher risk of tumor recurrence than lung cancer only in the univariate analyses (overall $\mathrm{p}=0.012, \mathrm{HR} 2.809$ [95\% CI 1.317-5.990], $\mathrm{p}=0.008$ and HR 2.292 [95\% CI 1.050-1.883], $\mathrm{p}=0.037$, respectively).

\section{Illustrative case}

A 73-year-old male patient with non-small cell lung cancer was diagnosed with a $2.9 \mathrm{~cm}$ brain metastatic lesion in the left temporo-occipital junction (Fig. 5 a). GKS with an initial prescription dose of $20 \mathrm{~Gy}$ at $50 \%$ IDL was performed. Due to suspicion of tumor progression at 11 months after the first session, a secondary prescription dose of 17 Gy at 50\% IDL was administered (Fig. 5b, IDL of the second GKS [yellow] and previous session [blue]). At 9 months from the last GKS, the patient visited the emergency room with IICP signs, and MRI showed a lesion resembling TRC with no distinct increase in CBV and absence of restricted diffusion (Fig. 5 c-e). Surgical resection was conducted, and the IICP improved. The pathology was confirmed to be a pure metastatic tumor cells (Fig 6). Without adjuvant local therapy for the resected lesion, the patient was free of local progression for 15 months (Fig 5f). 


\section{Discussion}

In this study, we investigated the clinical implication of TRC -PD representing patients with discrepant radiopathology and surgical histopathology. Despite heavy reliance on conventional imaging for progression guidelines and attention to subtleties in appearance with postcontrast $\mathrm{T} 1$ and FLAIR imaging, the sensitivity and specificity of standard MRI for detection of progression is low. Recently, a meta-analysis was conducted by Chuang et al. to investigate the utility of PWI and magnetic resonance spectroscopy (MRS) for differentiating recurrent tumors from necrosis in patients with various brain tumors ${ }^{10}$. Of the 397 patients in 13 studies, 95 patients had BM. The meta-analysis revealed that the $\mathrm{rCBV}$ derived from PWI, as well as MRS metabolite ratios in contrast-enhancing lesions, were significantly different in local progression compared with lesions resulting from radiation-related changes. Previous studies also reported variable diagnostic performance of PWI in terms of sensitivity (range: 56-100\%), specificity (range: 68-100\%), and relative CBV threshold (range: $1.52-2.14)^{7,18}$. Although many previous studies have tried a quantitative approach to PWI, the universal threshold for differentiating the tumor progression is not yet established and has low reproducibility. A recent survey indicated that approximately $50 \%$ of surveyed institutions do not process quantitative parameter maps due to high clinical cost-to-benefit ratio ${ }^{19}$.

MRS appears to provide high specificity for detecting tumor recurrence (almost 100\%). However, the relatively low sensitivity (range: $33-50 \%)$ and limited application for small-sized lesions $\left(<2 \mathrm{~cm}^{3}\right)$ are its two major drawbacks ${ }^{7,20}$. The potential benefit of amino acid positron emission tomography for differentiating pseudoprogression or $\mathrm{RN}$ from true disease progression following checkpoint inhibitor treatment of BM has been suggested, although the evidence is preliminary ${ }^{21}$.

In our study, most TRC or PD lesions, which worsened neurological deficits, were usually large with a median volume of $6.41 \mathrm{~cm}^{3}$ (range: $0.14-62.29 \mathrm{~cm}^{3}$ ). Thus, we primarily utilized only PWI and DWI plus standard MRI protocols for preoperative imaging to achieve diagnostic accuracy and timecost benefit in terms of determining urgent surgical resection.

With regard to clinical characteristics and prognosis, the TRC -PD group and TRC -RN or PD-PD groups were compared. The patients in the TRC -PD group had a larger PIV (median $9.40 \mathrm{~cm}^{3}$ ) than 
patients in the TRC -RN group (median $4.06 \mathrm{~cm}^{3}$ ), with no differences in the median prescription dose of 18 Gy (range: 14-25 Gy in TRC -RN and 8-22 Gy in TRC -PD). On deriving the median diameter from the median PIV in our study, the median target size values for the TRC -RN and TRC PD groups were $15.95 \mathrm{~mm}$ and $21.1 \mathrm{~mm}$, respectively. For targets in this range, a marginal dose of 21-24 Gy, or at least >18 Gy, has been suggested for local control according to previous studies ${ }^{22-24}$. Additionally, Amsbaugh et al. demonstrated that a unit increase in the maximum dose (Gy) per target size $(\mathrm{mm})$ was associated with a decreased local failure in SRS for BM, with the requirement for a higher prescription dose being proportional to the target size ${ }^{25}$. This may explain the histologic local failure of TRC-PD compared with TRC-RN under the same marginal dose.

The TRC-PD group exhibited a shorter time (median: 4.07 months) than the PD-PD group (median 8.77 months) from the last GKS session to the preoperative imaging diagnosis. Recent studies reported a local control rate of $87-93 \%$ at 6 months and $49-91 \%$ at 12 months in small-to-medium (1-3 cm)-sized BM lesions treated with $18 \mathrm{~Gy}{ }^{25,26}$. According to our results, early histologic local progression may have been overlooked in some patients due to the radiographic $\mathrm{RN}$ diagnosis. This would lead to a lower 6-month local control rate.

According to our survival analysis, the TRC-RN group appears to have a better OS than the TRCPD and PD-PD groups, although statistical significance was not observed. Histopathologic heterogeneity of the TRC-PD group, whether pure progressive metastatic tumor or a mixture of radiation necrosis and viable tumor cells, did not affect the survival or local progression. The risk of tumor recurrence after surgical resection was significantly higher in the PD-PD group alone than the other two groups in both the univariate and multivariate analyses. These results suggest a relatively benign prognosis of the TRC-PD group compared with that of the PD-PD group, regardless of the same histologic tumor progression. Rather it shows a benign prognosis of the "pure RN" (TRC-RN group), especially in terms of local recurrence.

This study has some limitations. It is a retrospective single-center study with a small cohort. Further radiomics-oriented analysis is required for a more precise differentiation of TRC and PD based on MR images, in terms of clinical prognosis. We did not include systemic therapies such as 
chemotherapy and immunotherapy, possible risk factors for $\mathrm{RN}$, due to the heterogeneity of individual therapeutic regimens.

In conclusion, notable histologic heterogeneity of metastatic lesions with TRC after radiosurgery was observed, represented as the TRC-PD group in this study. TRC may be pure RN or more frequently mixed pathology of necrosis and viable tumor tissue. This mixed RN with viable tumor cells (TRC-PD) had statistically equivalent benign prognosis as RN (TRC-RN), and more indolent than PD (PD-PD) after surgical resection. Surgical treatment is the feasible option for resolving the neurologic symptoms and archiving local control of TRCs after SRS in brain metastatic patients.

\section{Material and Methods}

\section{Study cohort}

The medical records of 201 patients who underwent surgical resection after GKS for BM at our institute between April 2009 and May 2019 were retrospectively reviewed. The Samsung Medical Center institutional review board approved this study and informed consent was waived by Young Keun On, MD., PhD. due to retrospective analyses (IRB number : 2020-11-147-001). All medical performance in this study were conducted according to current diagnostic or treatment guidelines. Patients with GKS dosimetry reports, advanced MRI sequences to discriminate TRCs and PD, and sufficient clinical follow-ups using MRI after surgery were included. We excluded patients with other surgical pathologies, acute intracranial hemorrhage or abscess, and preoperative GKS as adjuvant therapy. Patients who survived $<3$ months postoperatively were also excluded.

\section{Radiosurgical treatment}

Radiosurgery was performed with the Leksell Gamma Knife ${ }^{\circledR}$ Perfexion ${ }^{\mathrm{TM}}$ from 2009 to 2015 or the Leksell Gamma Knife ${ }^{\circledR}$ Icon $^{\mathrm{TM}}$ (both Elekta AB, Stockholm, Sweden) from April 2016 onward. The 1.0-mm slices of T1-weighted and 2.0-mm slices of T2-weighted fluid-attenuated inversion recovery (FLAIR) contrast-enhanced MR images were obtained and transferred to the Leksell 
GammaPlan Software version 11.1.1 (Elekta AB). The target volume was defined as the contrastenhancing tumor volume, and the dosimetry planning was conducted in accordance with the Radiation Therapy Oncology Group 90-05 study guidelines ${ }^{27,28}$.

\section{MRI protocols and image diagnosis}

The patients were clinically followed-up and evaluated with MRI every 2-3 months after radiosurgery. Each patient was scanned by 3-T MRI using the brain tumor MRI protocol in our institution, including pre- and post-contrast 5-mm-thick axial spin echo T1-weighted imaging, 5-mmthick axial fat-suppressed echo T2-weighted imaging, 5-mm-thick axial FLAIR imaging, and diffusion weighted imaging (DWI), which was performed with spin echo using a b-value of 0 and $1000 \mathrm{~s} / \mathrm{mm}^{2}$ (repetition time/echo time $[\mathrm{TR} / \mathrm{TE}]=3000 / 80 \mathrm{~ms}$, slice thickness $5 \frac{1}{4} \mathrm{~mm}$, interslice gap $=1.5 \mathrm{~mm}$, and acquisition matrix $=128 \times 128$ ), dynamic susceptibility contrast (DSC)-perfusionweighted imaging $(\mathrm{PWI})\left(\mathrm{TR} / \mathrm{TE}=1720 / 35 \mathrm{~ms}\right.$, flip angle $=40^{\circ}$, slice thickness $=5 \mathrm{~mm}$, interslice gap $=1.5 \mathrm{~mm}$, acquisition matrix $=128 \times 128,50$ volumes, and acquisition time $=1 \mathrm{~min} 30 \mathrm{~s}$ ). The amount of contrast agent was $0.1 \mathrm{mmol} / \mathrm{kg} \times 3 \mathrm{cc} / \mathrm{s}$ with power injector (Dotarem [gadoterate meglumine]; Guerbet, Aulnay-sous-Bois, France) in DSC-MRI. The maximum regional cerebral blood volume (rCBV) ratio of non-enhanced (NE) and contrast-enhanced (CE) portions, and minimum apparent diffusion coefficient of NE and CE portions on MRI were evaluated by drawing a region of interest circle with an area of $1 \mathrm{~cm}^{2}$. T2*-perfusion data were processed, and $\mathrm{rCBV}$ maps were computed using the software provided by the MR vendor (Intellispace Portal 9.0; Philips Health Tech, Best, the Netherlands) ${ }^{29,30}$.

Radiologic diagnoses were made by two experienced neuroradiologists (S.T.K. and J.S.R, both with more than 15-years of neuroradiologic practice). For post-GKS lesions that exhibited increased T1enhancement with T2/FLAIR hyperintensity suggesting the volumetric mass effect in follow-up MRI, tumor progression was suspected if a solid enhancement pattern, higher $\mathrm{rCBV}$, and more restricted diffusion were observed on qualitative inspection. TRCs were preferably diagnosed based on the "soap bubble" or "Swiss cheese" sign of peripheral enhancement, centrally necrotic lesions on T1- 
weighted post-contrast sequence, low or constant rCBV, and less restricted diffusion ${ }^{6,7,10,19,31}$.

\section{Indication for surgical resection and histopathologic diagnosis}

For patients who presented post-GKS neurological deficits or symptoms of increased intracranial pressure (IICP), treatment plan was thoroughly discussed on the Neuro-oncology conference. Symptomatic patients with imaging diagnoses of RN or PD were primarily treated with oral steroids. Thereafter, we conducted surgical resection of metastatic lesions if neurologic symptoms are refractory for medical treatment and patient's conditions are tolerable to surgery, of which Karnofsky performance scale (KPS) score is larger than 60 and life expectancy longer than 3 months.

Based on histopathological diagnosis, patients were classified into the following groups: PD group when their resected specimen showed prominent tumor cells $(>30 \%$ of the lesion composed of tumor cells); mixed $\mathrm{PD} / \mathrm{RN}$ group when their resected specimen showed predominant radiation-induced brain necrosis ( $>70 \%$ of the lesion) with viable tumor cells ( $<30 \%$ of the lesion); and $\mathrm{RN}$ group when no viable tumor cells were found in their specimens.

Patients underwent postoperative MRI within $48 \mathrm{~h}$ and routine outpatient follow-up 1 month after surgery and were further evaluated at 3-month intervals thereafter, and data were reported up to November 2020.

\section{Statistical analyses}

Patient demographics and clinical data were summarized using standard descriptive statistics and frequency tabulation. If statistically significant results indicated non-equal distribution, post hoc tests were used to identify significant differences between the groups. OS of patients was calculated from the time of resection until death or last follow-up. PFS was defined as the time from the surgical resection to the diagnosis of local progression of the resected lesion. Survival probability was estimated using the Kaplan-Meier product-limit method. Univariable and multivariable analyses were performed using a binary logistic regression model to identify the probability factors for local progression and survival. Statistical significance was determined when the p-value was $<0.05$. SAS 
version 9.4 (SAS Institute, Cary, NC) was used for all statistical analyses. 


\section{References}

1 Sperduto, P. W. et al. Effect of tumor subtype on survival and the graded prognostic assessment for patients with breast cancer and brain metastases. International Journal of Radiation Oncology* Biology* Physics 82, 2111-2117 (2012).

2 Shin, S. M. et al. Survival but not brain metastasis response relates to lung cancer mutation status after radiosurgery. Journal of neuro-oncology 126, 483-491 (2016).

3 Suh, J. H. Stereotactic radiosurgery for the management of brain metastases. New England Journal of Medicine 362, 1119-1127 (2010).

4 Gerosa, M. et al. Gamma knife radiosurgery for brain metastases: a primary therapeutic option. J Neurosurg 97, 515-524 (2002).

5 Petrovich, Z., Yu, C., Giannotta, S., O'Day, S. \& Apuzzo, M. Survival and pattern of failure in brain metastasis treated with stereotactic gamma knife radiosurgery. $J$ Neurosurg 97, 499-506 (2002).

6 Chao, S. et al. Challenges with the diagnosis and treatment of cerebral radiation necrosis. Int $J$ Radiat Oncol Biol Phys 87, 449-457 (2013).

7 Huang, J. et al. Differentiation between intra-axial metastatic tumor progression and radiation injury following fractionated radiation therapy or stereotactic radiosurgery using MR spectroscopy, perfusion MR imaging or volume progression modeling. Magnetic resonance imaging 29, 993-1001 (2011).

8 Artzi, M. et al. Differentiation between treatment-related changes and progressive disease in patients with high grade brain tumors using support vector machine classification based on DCE MRI. Journal of Neuro-Oncology 127, 515-524, doi:10.1007/s11060-016-2055-7 (2016).

9 Mitsuya, K. et al. Perfusion weighted magnetic resonance imaging to distinguish the recurrence of metastatic brain tumors from radiation necrosis after stereotactic radiosurgery. Journal of neuro-oncology 99, 81-88 (2010).

10 Chuang, M. T., Liu, Y. S., Tsai, Y. S., Chen, Y. C. \& Wang, C. K. Differentiating RadiationInduced Necrosis from Recurrent Brain Tumor Using MR Perfusion and Spectroscopy: A MetaAnalysis. PLoS One 11, e0141438, doi:10.1371/journal.pone.0141438 (2016).

11 Winter, S. F. et al. Defining Treatment-Related Adverse Effects in Patients with Glioma: Distinctive Features of Pseudoprogression and Treatment-Induced Necrosis. The oncologist 25, e1221 (2020).

12 Tofilon, P. J. \& Fike, J. R. The radioresponse of the central nervous system: a dynamic process. Radiation research 153, 357-370 (2000).

13 Winter, S. F. et al. Treatment-induced brain tissue necrosis: a clinical challenge in neurooncology. Neuro-oncology 21, 1118-1130 (2019). 
14 Dietrich, J., Winter, S. F. \& Klein, J. P. in Seminars in neurology. 589-596 (Thieme Medical Publishers).

15 Truong, M. T. et al. Results of surgical resection for progression of brain metastases previously treated by gamma knife radiosurgery. Neurosurgery 59, 86-97; discussion 86-97, doi:10.1227/01.Neu.0000219858.80351.38 (2006).

16 Tye, K. et al. An analysis of radiation necrosis of the central nervous system treated with bevacizumab. Journal of neuro-oncology 117, 321-327 (2014).

17 Dietrich, J., Rao, K., Pastorino, S. \& Kesari, S. Corticosteroids in brain cancer patients: benefits and pitfalls. Expert review of clinical pharmacology 4, 233-242 (2011).

18 Hatzoglou, V. et al. A prospective trial of dynamic contrast-enhanced MRI perfusion and fluorine-18 FDG PET-CT in differentiating brain tumor progression from radiation injury after cranial irradiation. Neuro-oncology 18, 873-880 (2016).

19 Kerkhof, M. et al. Clinical applicability of and changes in perfusion MR imaging in brain metastases after stereotactic radiotherapy. Journal of neuro-oncology 138, 133-139 (2018).

20 Chernov, M. F. et al. Multivoxel proton MRS for differentiation of radiation-induced necrosis and tumor recurrence after gamma knife radiosurgery for brain metastases. Brain tumor pathology 23, 19-27 (2006).

21 Galldiks, N. et al. PET imaging in patients with brain metastasis-report of the RANO/PET group. Neuro Oncol 21, 585-595, doi:10.1093/neuonc/noz003 (2019).

22 Wiggenraad, R. et al. Dose-effect relation in stereotactic radiotherapy for brain metastases. A systematic review. Radiotherapy and Oncology 98, 292-297 (2011).

23 Lippitz, B. et al. Stereotactic radiosurgery in the treatment of brain metastases: the current evidence. Cancer treatment reviews 40, 48-59 (2014).

24 Mohammadi, A. M. et al. Impact of the radiosurgery prescription dose on the local control of small (2 cm or smaller) brain metastases. Journal of Neurosurgery 126, 735-743 (2017).

25 Amsbaugh, M. et al. Dose-volume response relationship for brain metastases treated with frameless single-fraction linear accelerator-based stereotactic radiosurgery. Cureus 8 (2016).

26 Vogelbaum, M. A. et al. Local control of brain metastases by stereotactic radiosurgery in relation to dose to the tumor margin. Journal of neurosurgery 104, 907-912 (2006).

27 Cho, K. R. et al. Outcome of gamma knife radiosurgery for metastatic brain tumors derived from non-small cell lung cancer. $J$ Neurooncol 125, 331-338, doi:10.1007/s11060-015-1915-x (2015).

28 Jeon, C. et al. Outcome of three-fraction gamma knife radiosurgery for brain metastases according to fractionation scheme: preliminary results. Journal of Neuro-Oncology 145, 65-74, doi:10.1007/s11060-019-03267-z (2019).

29 Seong, M., Kim, S. T., Noh, J. H., Kim, Y. K. \& Kim, H. J. Radiologic findings and the 
molecular expression profile of diffuse midline glioma H3 K27M mutant. Acta Radiol, 284185120968560, doi:10.1177/0284185120968560 (2020).

30 Seong, M. et al. Diagnostic accuracy of MR imaging of patients with leptomeningeal seeding from lung adenocarcinoma based on 2017 RANO proposal: added value of contrast-enhanced 2D axial T2 FLAIR. Journal of Neuro-Oncology 149, 367-372, doi:10.1007/s11060-02003617-2 (2020).

31 Walker, A. J. et al. Postradiation imaging changes in the CNS: how can we differentiate between treatment effect and disease progression? Future Oncol 10, 1277-1297, doi:10.2217/fon.13.271 (2014). 
Funding: This research did not receive any specific grant from funding agencies in the public, commercial, or not-for-profit sectors.

\section{Author Contributions}

Jeong Hwa Kim: Conceptualization, Data curation, Formal Analysis, Writing - Original Draft, Writing - Review and Editing;

Jung Won Choi: Conceptualization, Data Curation, Supervision - Review and Editing;

Doo-Sik Kong: Data Curation, Supervision, Writing - Review and Editing;

Ho Jun Seol: Data Curation, Supervision, Writing - Review and Editing;

Do-Hyun Nam: Data Curation, Supervision, Writing - Review and Editing;

Jae-Wook Ryu: Conceptualization, Data Curation - Review and Editing;

Sung-Tae Kim: Conceptualization, Data Curation, Formal Analysis - Review and Editing;

Yeon-Lim Suh: Formal Analysis, Writing - Review and Editing;

Jung-Il Lee: Conceptualization, Data Curation, Formal Analysis, Supervision, Writing - Review and Editing.

Conflicts of interest/Competing interests: The authors report no conflict of interest concerning the materials or methods used in this study or the findings specified in this paper. 


\section{Figure Captions}

Fig. 1 Flow chart of patient enrollment and classification. Eighty-six patients were included and classified into four groups according to preoperative MRI and surgical pathology. For surgical pathology, pure radiation necrosis alone was sorted into "radiation necrosis." Mixed pathology, containing both metastatic tumor and necrotic cells, was classified as "metastatic cells." No patient was assigned to the PD-RN group; therefore, the three groups TRC-RN, TRC-PD, and PD-PD were finally incorporated in this study.

$\mathrm{MRI}=$ magnetic resonance imaging $; \mathrm{PD}=$ progressive disease $; \mathrm{RN}=$ radiation necrosis $; \mathrm{TRC}=$ treatment-related change.

Fig. 2 Kaplan-Meier estimate of overall survival

Fig. 3 Kaplan-Meier estimate of progression-free survival

Fig. 4 Subgroup analysis of patients with TRC-PD. (a) (b) Comparison of the KM curve of OS and PFS between TRC-PD patients with pure metastatic tumor cells $(n=24)$ and mixed pathology of radiation necrosis and progressive tumor $(\mathrm{n}=11)$, respectively.

$\mathrm{OS}=$ overall survival $; \mathrm{PD}=$ progressive disease $; \mathrm{PFS}=$ progression-free survival; $\mathrm{RN}=$ radiation necrosis; $\mathrm{TRC}=$ treatment-related change.

Fig. 5 An illustrative case of TRC-PD

$\mathrm{PD}=$ progressive disease; $\mathrm{TRC}=$ treatment-related image change.

Fig. 6. Representative histopathologic features of patients with TRC-PD with mixed pathology of PD and $\mathrm{RN}$

The pathology was consistent with that of the illustrative case shown in Figure 5. Adjacent to a large, 
confluent necrotic portion (Right), an area with viable tumor cells (Left) was present, rendering a diagnosis of metastatic adenocarcinoma. (hematoxylin and eosin stain, $\times 100$, scale bar $=500 \mu \mathrm{m}$ ) $\mathrm{PD}=$ progressive disease $; \mathrm{RN}=$ radiation necrosis $; \mathrm{TRC}=$ treatment-related change 
Table 1. Patient demographics and treatment parameters

\begin{tabular}{|c|c|c|c|c|c|}
\hline & $\begin{array}{l}\text { Total } \\
(n=86)\end{array}$ & $\begin{array}{l}\text { TRC-RN } \\
(n=19)\end{array}$ & $\begin{array}{l}\text { TRC-PD } \\
(n=35)\end{array}$ & $\begin{array}{l}\text { PD-PD } \\
(n=32)\end{array}$ & p-value \\
\hline Median age, year (range) & $58(40-81)$ & $57(40-77)$ & $58(40-74)$ & $58(41-81)$ & 0.943 \\
\hline $\operatorname{Sex}(\%)$ & & & & & 0.879 \\
\hline Male & $36(41.9)$ & $7(36.8)$ & $15(42.9)$ & $14(43.8)$ & \\
\hline Female & $50(58.1)$ & $12(63.2)$ & $20(57.1)$ & $18(56.2)$ & \\
\hline Primary malignancy (\%) & & & & & 0.169 \\
\hline Lung & $57(66.3)$ & $14(73.7)$ & $27(77.1)$ & $16(50.0)$ & \\
\hline Breast & $15(17.4)$ & $2(10.5)$ & $5(14.3)$ & $8(25.0)$ & \\
\hline${ }^{b}$ Other & $14(16.3)$ & $3(15.8)$ & $3(8.6)$ & $8(25.0)$ & \\
\hline $\begin{array}{l}\text { Multiple GKS history for surgically } \\
\text { resected lesion }(\%)\end{array}$ & & & & & $0.005^{*}$ \\
\hline 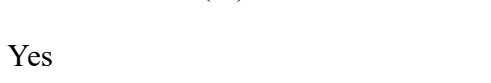 & $28(32.6)$ & $12(63.2)$ & $9(25.7)$ & $7(21.9)$ & $\begin{array}{l}-2.68^{a} \\
0.007^{a}\end{array}$ \\
\hline No & $58(67.4)$ & $7(36.8)$ & $26(74.3)$ & $25(78.1)$ & \\
\hline Prior WBRT (\%) & & & & & 0.117 \\
\hline Yes & $18(20.9)$ & $7(36.8)$ & $7(20.0)$ & $4(12.5)$ & \\
\hline No & $68(79.1)$ & $12(63.2)$ & $28(80.0)$ & $28(87.5)$ & \\
\hline Location (\%) & & & & & 0.754 \\
\hline Frontal & $40(46.5)$ & $8(42.1)$ & 17 (48.6) & $15(46.9)$ & \\
\hline Parietal & $22(25.6)$ & $6(31.5)$ & $10(28.5)$ & $6(18.7)$ & \\
\hline Temporal & $5(5.8)$ & $1(5.3)$ & $1(2.9)$ & $3(9.4)$ & \\
\hline Occipital & $7(8.1)$ & $1(5.3)$ & $2(5.7)$ & $4(12.5)$ & \\
\hline Cerebellum & $11(12.8)$ & $2(10.5)$ & $5(14.3)$ & $4(12.5)$ & \\
\hline Other & $1(1.2)$ & $1(5.3)$ & $0(0)$ & $0(0)$ & \\
\hline Median PIV, $\mathrm{cm}^{3}$ (range) & $\begin{array}{l}6.41 \\
(0.14-62.29)\end{array}$ & $\begin{array}{l}4.06 \\
(0.14-34.50)\end{array}$ & $\begin{array}{l}9.40 \\
(1.50-40.72)\end{array}$ & $\begin{array}{l}6.80 \\
(0.89-62.29)\end{array}$ & $0.014^{*}$ \\
\hline Median prescription dose, Gy (range) & $18(8-25)$ & $18(14-25)$ & $18(8-22)$ & $17(12-23)$ & 0.605 \\
\hline $\begin{array}{l}\text { Median time interval from GKS to } \\
\text { imaging diagnosis of TRC or PD, } \\
\text { months (range) }\end{array}$ & $\begin{array}{l}6.07 \\
(3.01-40.03)\end{array}$ & $\begin{array}{l}5.43 \\
(3.01-39.60)\end{array}$ & $\begin{array}{l}4.07 \\
(3.40-18.13)\end{array}$ & $\begin{array}{l}8.77 \\
(4.10-40.03)\end{array}$ & $0.004 *$ \\
\hline Primary malignancy control (\%) & & & & & 0.413 \\
\hline Controlled & $41(47.7)$ & $9(47.4)$ & $14(40.0)$ & $18(56.3)$ & \\
\hline Uncontrolled & $45(52.3)$ & $10(52.7)$ & $21(60.0)$ & $14(43.8)$ & \\
\hline
\end{tabular}


*overall p-value

${ }^{\mathrm{a}} \mathrm{z}$ - and p-values of the Jonckheere-Terpstra test

${ }^{b}$ renal cell cancer (3), colorectal cancer (4), melanoma (2), hepatocellular carcinoma (2), cholangiocarcinoma, endometrial carcinoma, thyroid cancer

$\mathrm{GKS}=$ gamma knife radiosurgery; $\mathrm{PD}=$ progressive disease; $\mathrm{PIV}=$ prescription isodose volume $\mathrm{RN}=$ radiation necrosis; 
Table 2. Univariate and multivariate analyses for risk of death and tumor recurrence

\begin{tabular}{|c|c|c|c|c|c|c|c|c|c|}
\hline \multirow{3}{*}{\begin{tabular}{|l} 
Continuous data \\
\end{tabular}} & & \multicolumn{4}{|c|}{ Overall survival } & \multicolumn{4}{|c|}{ Progression-free survival } \\
\hline & & \multicolumn{2}{|c|}{ Univariate analysis } & \multicolumn{2}{|c|}{ Multivariate analysis } & \multicolumn{2}{|c|}{ Univariate analysis } & \multicolumn{2}{|c|}{ Multivariate analysis } \\
\hline & & HR (95\% CI) & p-value & HR $(95 \%$ CI) & p-value & HR (95\% CI) & p-value & HR (95\% CI) & p-value \\
\hline Age, years & & $1.004(0.974-1.035)$ & 0.801 & & & $1.023(0.990-1.057)$ & 0.154 & & \\
\hline $\mathrm{PIV}, \mathrm{mm}^{3}$ & & $0.992(0.967-1.017)$ & 0.519 & $0.971(0.944-1.000)$ & 0.048 & $1.007(0.981-1.032)$ & 0.615 & $1.001(0.973-1.031)$ & 0.925 \\
\hline Prescription dose, G. & & $0.941(0.852-1.040)$ & 0.234 & & & $0.887(0.796-0.989)$ & 0.030 & $0.912(0.778-1.067)$ & 0.250 \\
\hline $\begin{array}{l}\text { Time interval GKS - } \\
\text { months }\end{array}$ & TRC or PD, & $0.966(0.930-1.003)$ & 0.069 & $0.961(0.921-1.003)$ & 0.071 & $0.999(0.968-1.031)$ & 0.957 & $0.990(0.950-1.032)$ & 0.643 \\
\hline \multicolumn{10}{|l|}{ Categorical data } \\
\hline TRC-RN & & 1 & $0.757^{*}$ & 1 & $0.055^{*}$ & 1 & $<0.001^{*}$ & 1 & $0.002 *$ \\
\hline TRC-PD & & $2.313(1.008-5.308)$ & 0.048 & $3.342(1.153-9.692)$ & 0.026 & $1.116(0.432-2.881)$ & 0.821 & $1.412(0.474-4.204)$ & 0.535 \\
\hline PD-PD & & $2.469(1.100-5.543)$ & 0.028 & $3.063(1.125-8.345)$ & 0.026 & $4.109(1.725-9.788)$ & 0.001 & $5.461(1.857-16.061)$ & 0.002 \\
\hline Sex & $\begin{array}{l}\text { Male } \\
\text { Female }\end{array}$ & $\begin{array}{l}1 \\
0.736(0.414-1.310)\end{array}$ & 0.298 & & & $\begin{array}{l}1 \\
1.143(0.604-2.162)\end{array}$ & 0.681 & & \\
\hline $\begin{array}{l}\text { Primary } \\
\text { malignancy }\end{array}$ & $\begin{array}{l}\text { Lung } \\
\text { Breast } \\
\text { Other }\end{array}$ & $\begin{array}{l}1 \\
1.296(0.610-2.753) \\
2.305(1.165-4.559)\end{array}$ & $\begin{array}{c}0.056^{*} \\
0.500 \\
0.017\end{array}$ & $\begin{array}{l}1 \\
0.940(0.427-2.071) \\
1.874(0.866-4.055)\end{array}$ & $\begin{array}{c}0.216^{*} \\
0.878 \\
0.111\end{array}$ & $\begin{array}{l}1 \\
2.809(1.317-5.990) \\
2.292(1.050-5.002)\end{array}$ & $\begin{array}{l}0.012^{*} \\
0.008 \\
0.037\end{array}$ & $\begin{array}{l}1 \\
1.987(0.830-4.760) \\
1.286(0.535-3.090)\end{array}$ & $\begin{array}{l}0.305^{*} \\
0.123 \\
0.574\end{array}$ \\
\hline Prior GKS & $\begin{array}{l}\text { No } \\
\text { Yes }\end{array}$ & $\begin{array}{l}1 \\
0.715(0.379-1.379)\end{array}$ & 0.300 & $\begin{array}{l}1 \\
0.796(0.378-1.676)\end{array}$ & 0.548 & $\begin{array}{l}1 \\
0.974(0.504-1.883)\end{array}$ & 0.938 & $\begin{array}{l}1 \\
1.060(0.431-2.607)\end{array}$ & 0.900 \\
\hline WBRT & $\begin{array}{l}\text { No } \\
\text { Yes }\end{array}$ & $\begin{array}{l}1 \\
0.890(0.453-1.751)\end{array}$ & 0.736 & & & $\begin{array}{l}1 \\
0.633(0.280-1.431)\end{array}$ & 0.272 & & \\
\hline $\begin{array}{l}\text { Primary } \\
\text { malignancy control }\end{array}$ & $\begin{array}{l}\text { Controlled } \\
\text { Uncontrolled }\end{array}$ & $\begin{array}{l}1 \\
2.027(1.143-3.595)\end{array}$ & 0.016 & $\begin{array}{l}1 \\
1.743(0.849-3.579)\end{array}$ & 0.130 & $\begin{array}{l}1 \\
1.420(0.762-2.647)\end{array}$ & 0.269 & & \\
\hline
\end{tabular}


*overall p-value, statistical significance, $\mathrm{p}<0.05$

$\mathrm{CI}=$ confidence interval; $\mathrm{GKS}=$ gamma knife radiosurgery; $\mathrm{HR}=$ hazard ratio $\mathrm{NA}=$ not included in the analysis; $\mathrm{PD}=$ progressive disease; $\mathrm{PIV}=$ prescription isodose volume; $\mathrm{RN}=$ radiation necrosis; $\mathrm{TRC}=$ treatment-related change; WBRT $=$ whole-brain radiation therapy. 


\section{Figures}

Patients who underwent surgical resection for brain metastases

after GKS between April 2009 to May 2019 (n=201)

\section{Excluded ( $n=115)$}

- Planned surgery with preoperative GKS $(n=35)$

- Insufficient preoperative imaging to differentiate between TRC and PD ( $n=26)$

- Surgical pathologies except RN or PD (other tumor pathology, intracranial hemorrhage, radiation-induced cavernoma, and abscess etc.) $(n=25)$

- Follow-up loss after surgery $(n=22)$

- Postoperative clinical follow-up less than 3 months $(\mathrm{n}=7)$

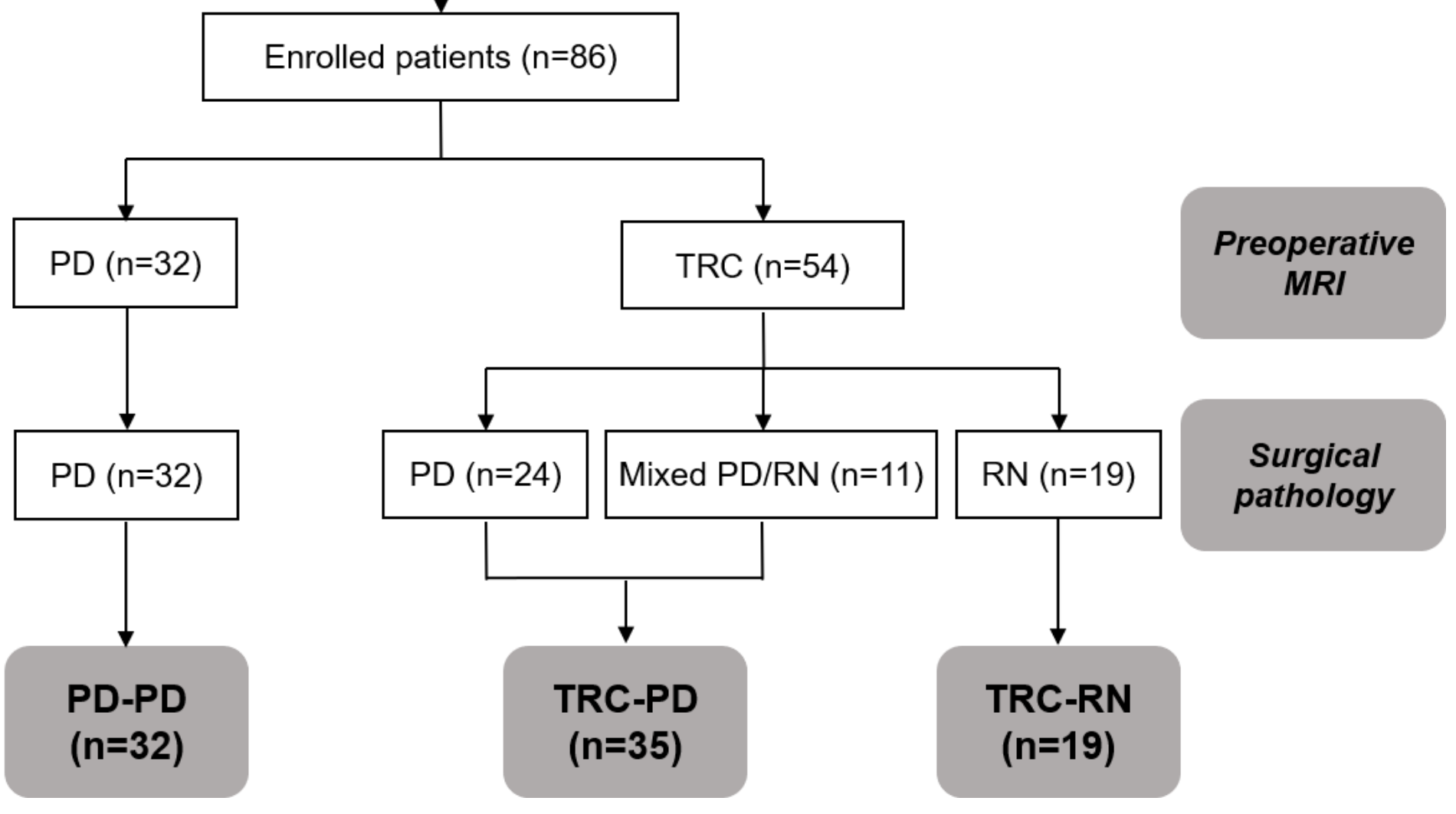

Figure 1

Flow chart of patient enrollment and classification. Eighty-six patients were included and classified into four groups according to preoperative MRI and surgical pathology. For surgical pathology, pure radiation necrosis alone was sorted into "radiation necrosis." Mixed pathology, containing both metastatic tumor 
and necrotic cells, was classified as "metastatic cells." No patient was assigned to the PD-RN group; therefore, the three groups TRC-RN, TRC-PD, and PD-PD were finally incorporated in this study. MRI = magnetic resonance imaging; $\mathrm{PD}=$ progressive disease; $\mathrm{RN}=$ radiation necrosis; $\mathrm{TRC}$ = treatment-related change.

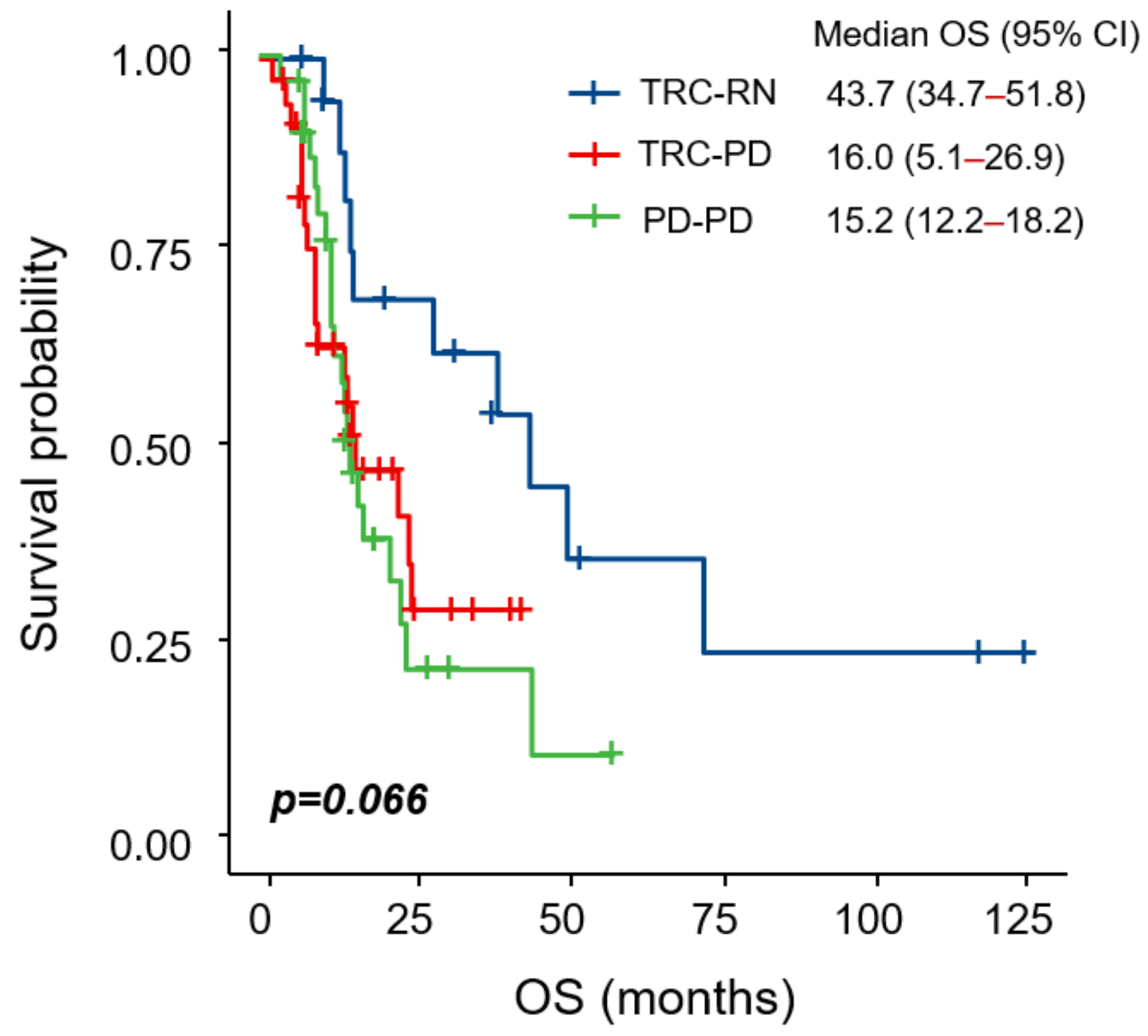

Number at risk

\begin{tabular}{r|llllll} 
TRC-RN & 19 & 10 & 4 & 2 & 2 & 1 \\
TRC-PD & 35 & 7 & 0 & 0 & 0 & 0 \\
PD-PD & 32 & 4 & 1 & 0 & 0 & 0 \\
\cline { 2 - 8 }
\end{tabular}

Figure 2

Kaplan-Meier estimate of overall survival 


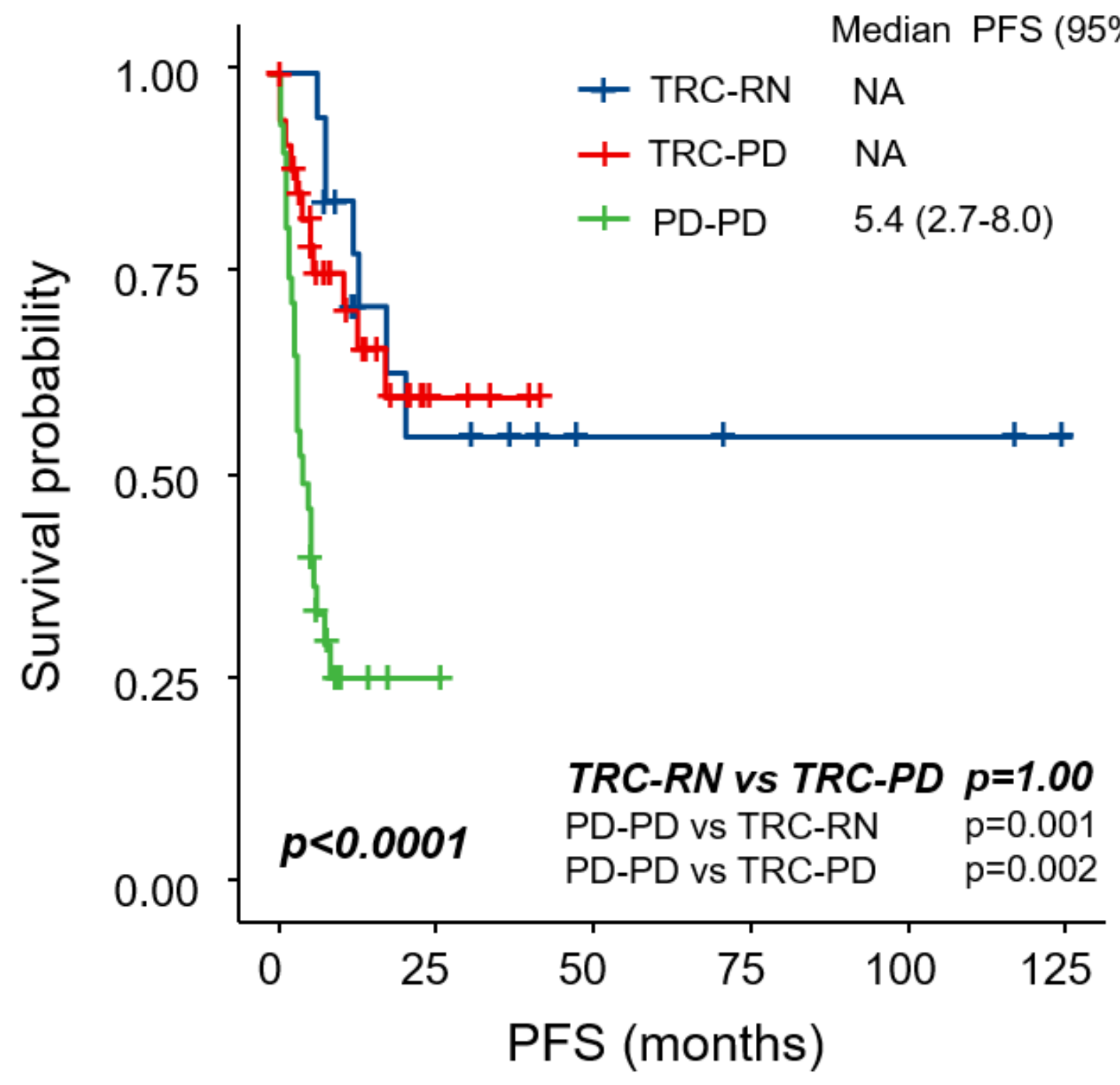

Number at risk

\begin{tabular}{r|rrrrrr} 
TRC-RN & 19 & 7 & 3 & 2 & 2 & 1 \\
TRC-PD & 35 & 7 & 0 & 0 & 0 & 0 \\
PD-PD & 32 & 1 & 0 & 0 & 0 & 0 \\
\hline
\end{tabular}

Figure 3

Kaplan-Meier estimate of progression-free survival 


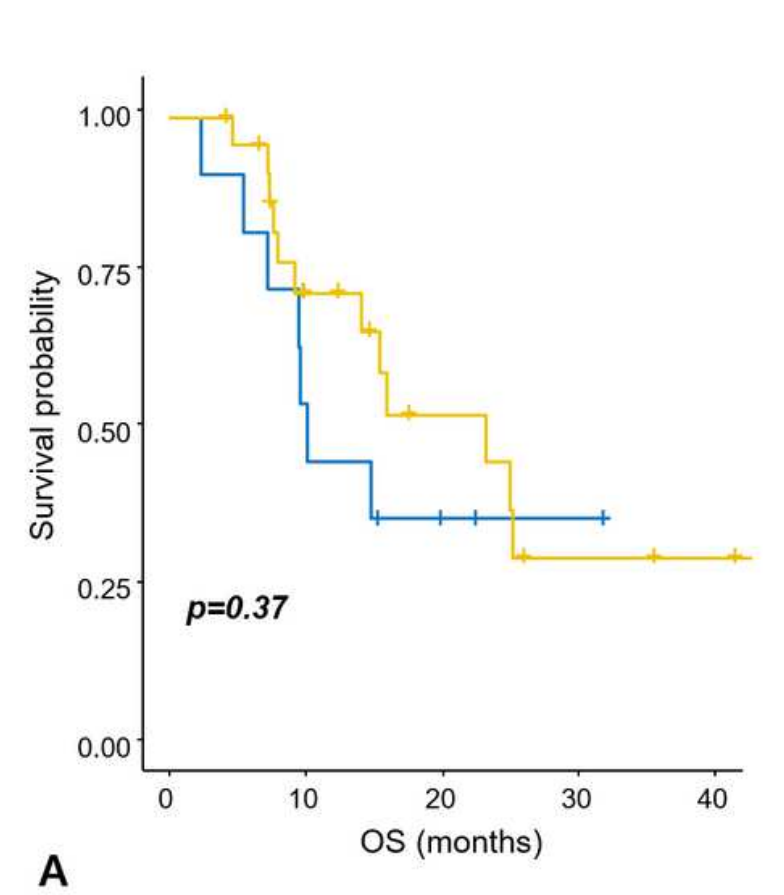

A

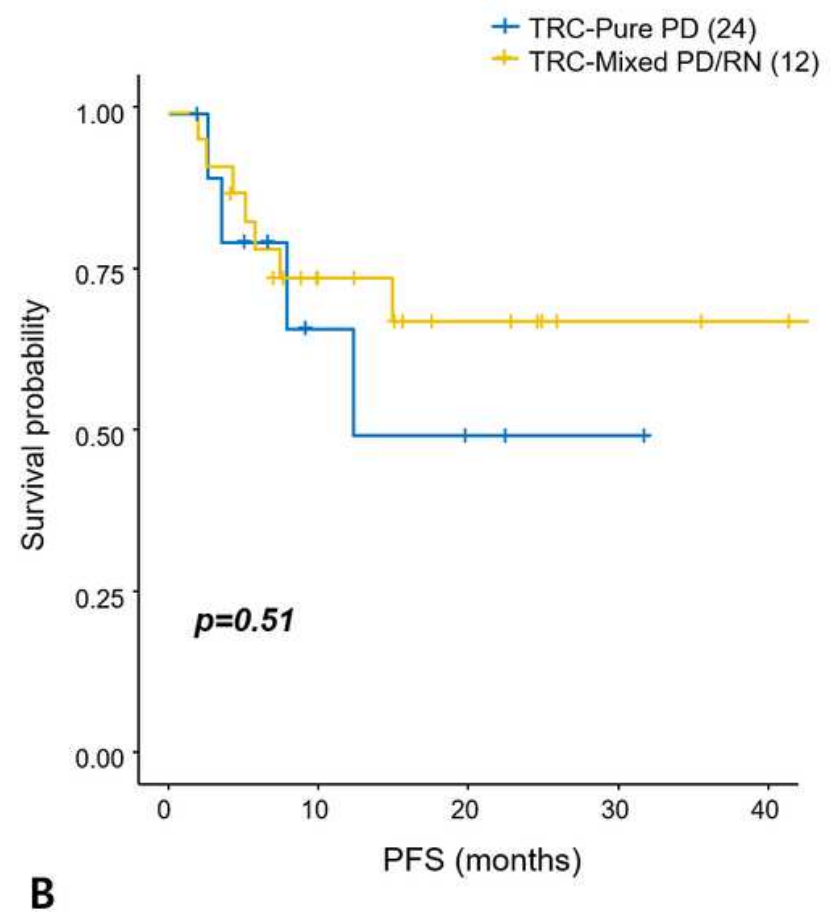

\section{Figure 4}

Subgroup analysis of patients with TRC-PD. (a) (b) Comparison of the KM curve of OS and PFS between TRC-PD patients with pure metastatic tumor cells $(n=24)$ and mixed pathology of radiation necrosis and progressive tumor $(n=11)$, respectively. $O S$ = overall survival; $P D=$ progressive disease; $P F S=$ progression-free survival; $\mathrm{RN}$ = radiation necrosis; $\mathrm{TRC}$ = treatment-related change. 


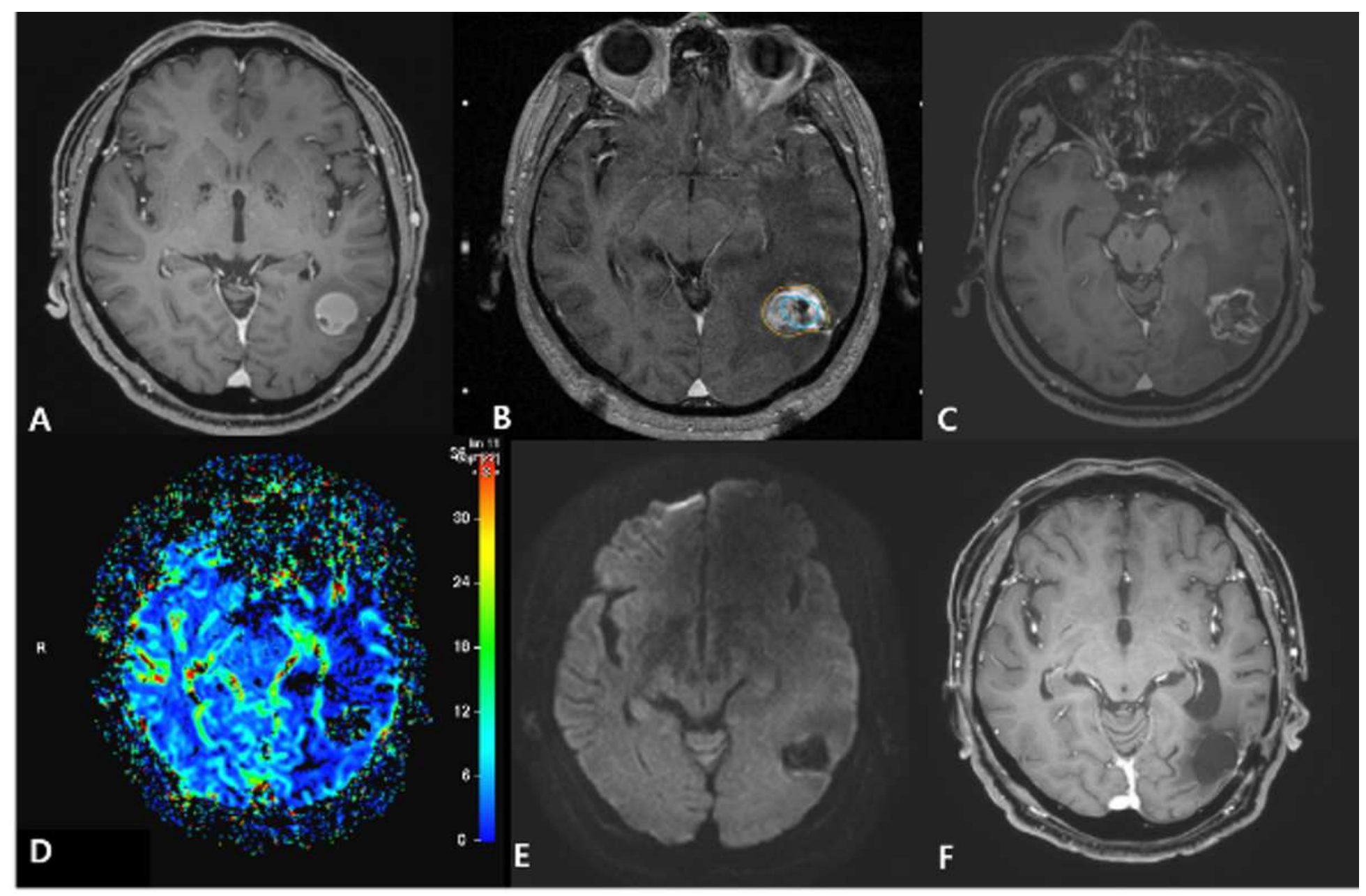

Figure 5

An illustrative case of TRC-PD PD = progressive disease; TRC = treatment-related image change. 


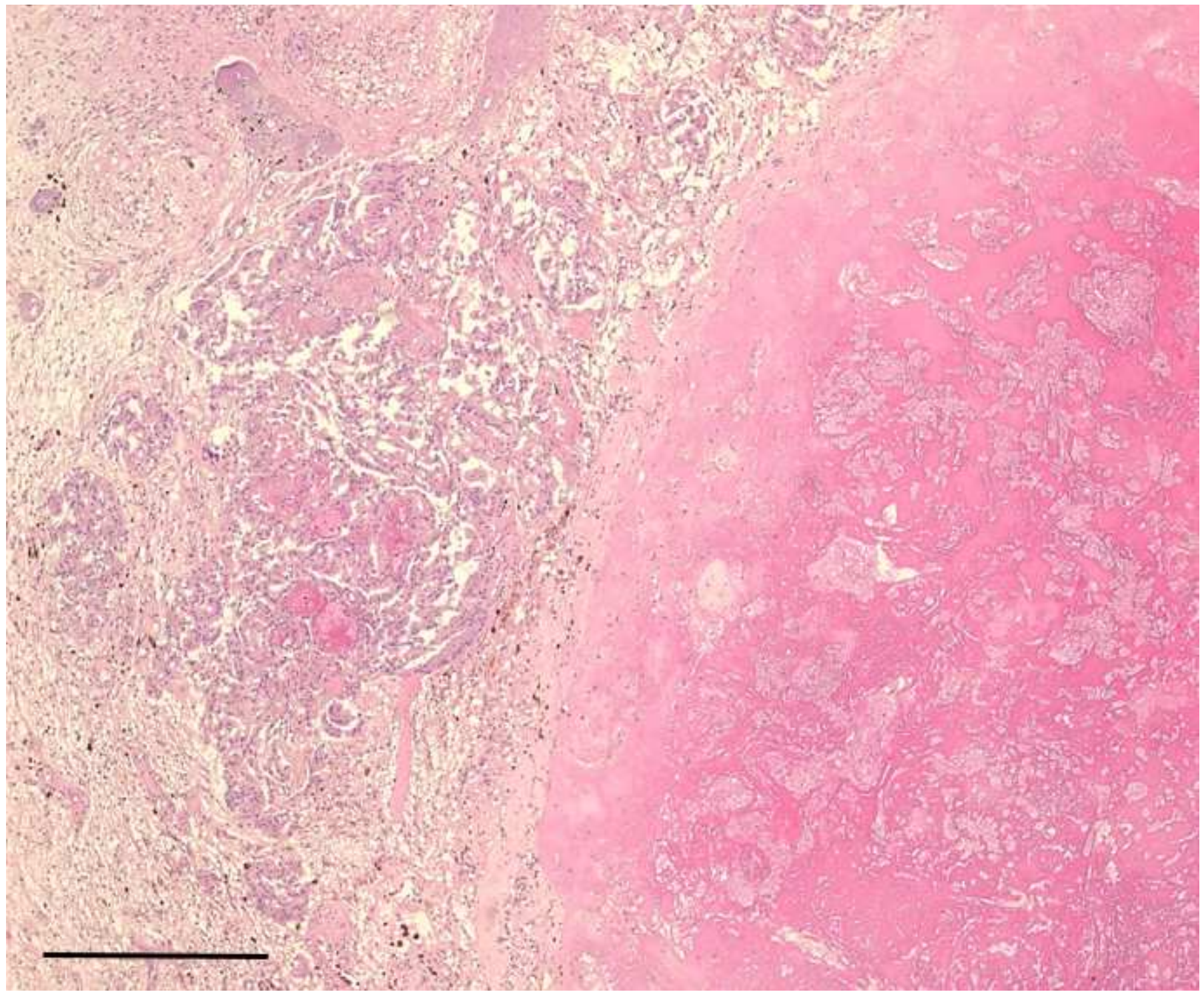

Figure 6

Representative histopathologic features of patients with TRC-PD with mixed pathology of PD and RN The pathology was consistent with that of the illustrative case shown in Figure 5. Adjacent to a large, confluent necrotic portion (Right), an area with viable tumor cells (Left) was present, rendering a diagnosis of metastatic adenocarcinoma. (hematoxylin and eosin stain, $\times 100$, scale bar $=500 \mu \mathrm{m}$ ) PD = progressive disease; $\mathrm{RN}=$ radiation necrosis; $\mathrm{TRC}$ = treatment-related change 